\title{
Laser-Plasma Accelerators: A Status Report
}

\author{
C. Joshi \\ UCLA, Los Angeles, CA 90095
}

\section{INTRODUCTION}

In this paper, the status of the Laser-Plasma Accelerator field within the Advanced Accelerator Concepts area of research is reviewed. In particular, I review the status of the Plasma Beat Wave Acceleration ${ }^{1}$ (PBWA) scheme, the SelfModulated, Laser-Wake Field Acceleration ${ }^{2}$ (SMLWFA) scheme and the Laser-Wake Field Acceleration ${ }^{3}$ (LWFA) scheme. In all these three schemes, charged particles are accelerated by a relativistic (phase velocity $\sim c$ ), space-charge wave excited in a plasma by a photon beam. The wave potential becomes large enough to trap either the background plasma electrons, in a process known as self-trapping, ${ }^{4}$ or electrons (positrons) must be externally injected with a certain minimum energy ${ }^{5}$ to be trapped and accelerated by this potential. There is a simple scaling law that gives the accelerating electric field of such waves as $\mathrm{E}_{\mathrm{z}}(\mathrm{V} / \mathrm{cm}) \sim \varepsilon\left(\mathrm{n}_{\mathrm{e}}\right)^{1 / 2}\left(\mathrm{~cm}^{-3}\right)$ where $\varepsilon=\tilde{\mathrm{n}} / \mathrm{n}_{\mathrm{o}}$ is the density perturbation associated with the wave and $n_{e}$ is the initial unperturbed plasma density. The maximum energy gain limited by dephasing is given by $\sim 2 \gamma_{\mathrm{ph}}^{2}$ $(\mathrm{MeV})$ where $\gamma_{\mathrm{ph}}=\left(\omega_{\mathrm{o}} / \omega_{\mathrm{p}}\right)$ is the relativistic Lorentz factor associated with the phase velocity of the wave, $\omega_{0}$ is the laser frequency and $\omega_{\mathrm{p}}$ is the plasma frequency.

\section{STATUS REPORT ON THE PLASMA BEAT WAVE ACCELERATION (PBWA)}

In the PBWA, two co-propagating laser beams, with slightly different frequencies and wave numbers, beat-excite the relativistic plasma wave if the frequency difference $\Delta \omega=\omega_{1}-\omega_{2}$ is equal to the plasma frequency $\omega_{\mathrm{p}}$. In this case the wave number of the plasma wave is $\mathrm{k}_{\mathrm{p}}=\Delta \mathrm{k}=\mathrm{k}_{1}-\mathrm{k}_{2}$ and $\omega_{\mathrm{p}} / \mathrm{k}_{\mathrm{p}} \cong \mathrm{c}$ with $\gamma_{\mathrm{ph}}=\left(\omega_{1}\right.$ $\left.+\omega_{2}\right) / 2 \omega_{\mathrm{p}}$. The physical mechanism for displacing the plasma electrons from their initial position is the, so-called, ponderomotive force, which is proportional to the gradient of the dot product of the electric fields of the two lasers. Since beat excitation is a resonant process, the laser intensity required excite a large amplitude $(\varepsilon>0.1)$ plasma oscillation is relatively modest and the laser pulse can be fairly long. 
Characterizing the laser intensities and the pulse length in the normalized units of the vector potential $\mathrm{a}=\mathrm{eE} / \mathrm{mc} \omega$ and collisionless skin depth $\mathrm{c} / \omega_{\mathrm{p}}$ respectively, the PBWA typically requires $a_{1,2}=0.1$ and $\tau \cong 10-100 \mathrm{c} / \omega_{\mathrm{p}}$. This translates to $\mathrm{I}_{1,2}=10^{14} \mathrm{~W} / \mathrm{cm}^{2}$ and $\tau \cong 100 \mathrm{ps}$ for $\mathrm{a} \mathrm{CO}_{2}$ laser driver and $\gamma_{\mathrm{ph}} \cong 30$ plasma wave.

The PBWA scheme was anticipated in the original Laser Electron Accelerator ${ }^{3}$ paper of Tajima and J. M. Dawson. However, many scientists doubted whether such a scheme would work in practice because of the relatively long and intense pulses used to excite the plasma wave. There was worry that competing laser-plasma instabilities would "kill" the scheme. It was not until 1987 when first 2D, PIC code simulations of the PBWA scheme were carried out in the context of a particle accelerator that the potential of this method as an ultrahigh gradient particle accelerator became widely accepted. ${ }^{1}$ Figure 1 below shows one result from Reference (1) showing that there was a quantitative agreement between the growth rate of the plasma wave seen using PIC simulations and that predicted by the fluid theory developed by M. N. Rosenbluth and C. S. Liu. ${ }^{6}$
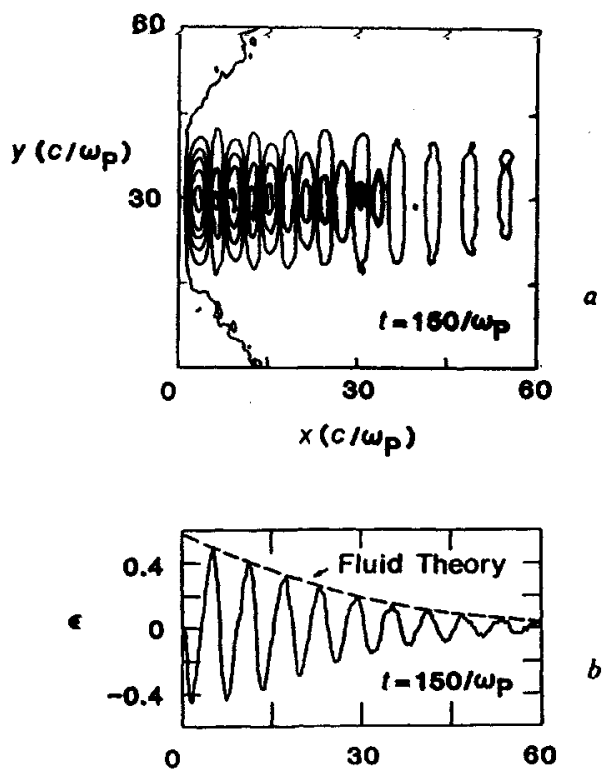

Figure 1. (a) $2 \mathrm{D}$ contours of the wave potential in space and (b) the growth of the plasma wave in time as predcted by 2D-PIC simulations of the PBWA (ref. 1).

Reference (1) was followed shortly by an experimental verification of the excitation of relativistic plasma waves by collinear optical mixing ${ }^{7}$ by the UCLA group. Figure 2 shows key results from this paper which demonstrated the frequency $\left(\omega_{p}=\Delta \omega\right)$ and wave number $\left(\mathrm{k}_{\mathrm{p}}=\Delta \mathrm{k}\right)$ relationship for the excited plasma wave; the excitation of the Stokes and anti-Stokes side bands and a proportionate relationship between the 
amplitude of the first Stokes side band and the amount of Thomson scattering from the plasma wave. It was this paper that gave credibility to the whole Plasma Accelerator field and resulted in many experiments being funded around the world on actual acceleration of electrons using such waves.
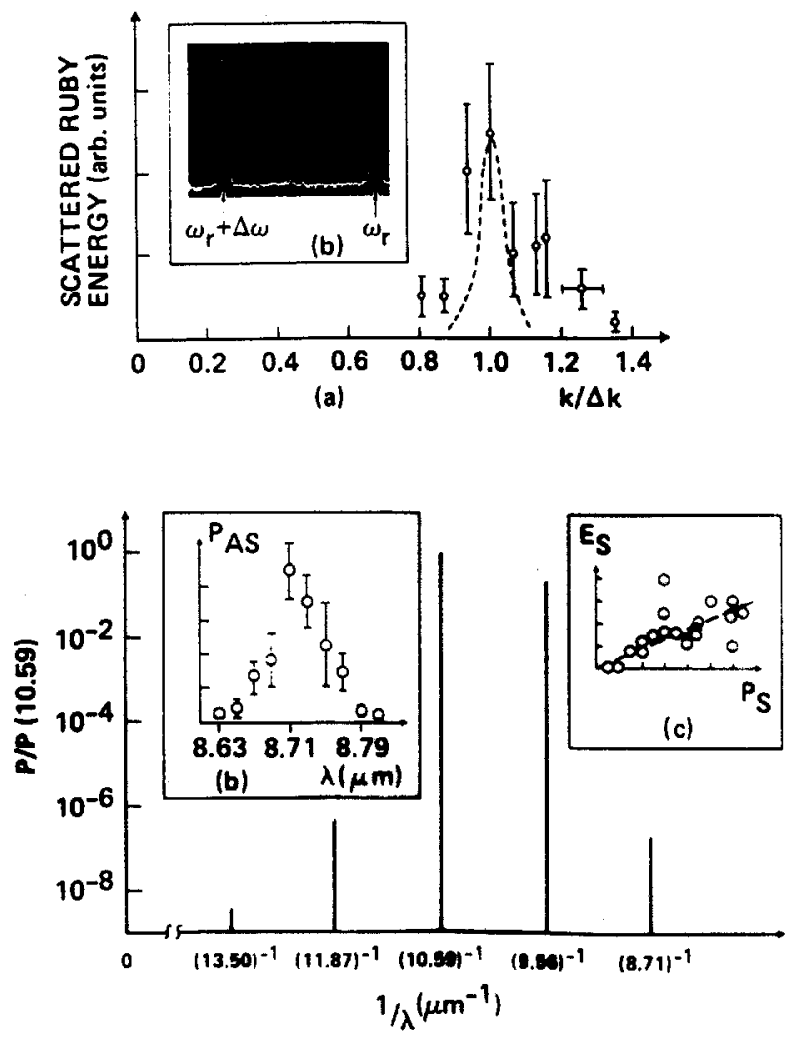

(a)

Figure 2. (a) The frequency and wavenumber spectrum of the beat-excited relativistic plasma wave, (b) the stokes and anti-stokes side bands including the anti-stokes spectrum (inset) and (c) linear relationship between thomson scattered light $\left(E_{s}\right)$ and stokes light $P_{s}(\operatorname{Ref} .7)$.

It was 1993 when first conclusive results were presented by the UCLA group of acceleration and substantial energy gain of externally injected electrons using the PBWA technique. ${ }^{8}$ Figure 3 shows key results from that publication. Many plasma physicists doubted that one could conclusively demonstrate acceleration of test particles in a violent plasma environment. So the UCLA group used first a monochrometer to momentum select electrons of a certain energy and then an auxiliary cloud chamber to visualize the particles. A secondary orthogonal B field was used to bend the electrons in the cloud chamber and measure their relativistic 
Larmor radius to confirm the energy gain. Futhermore, both the electron energy gain and the number of accelerated electrons were found to maximize at the resonant density. (In Fig. 3(b) the horizontal axis is pressure but later this was shown to be directly proportional to electron density).

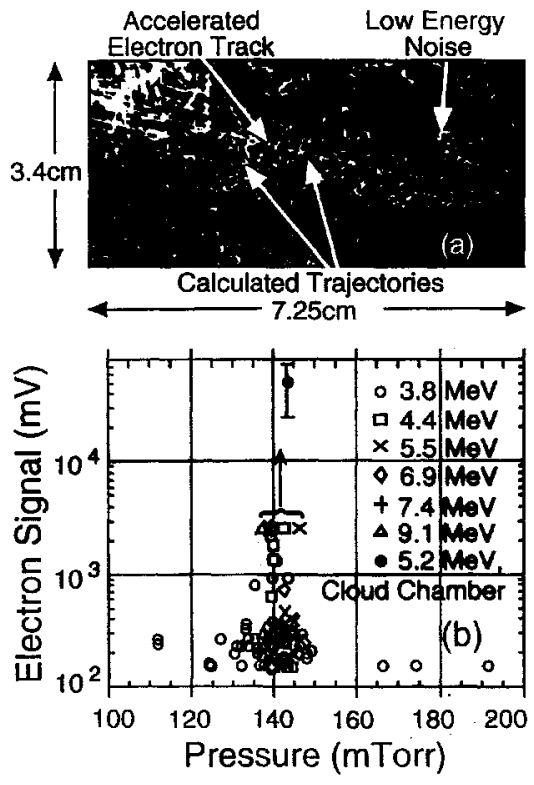

Figure 3. (a) Cloud chamber tracks produced by accelerated electrons and (b) the number of accelerated electron as the gas pressure (plasma density) is varied showing the expected resonance (Ref. 8).

In 1994, the UCLA group showed trapping and acceleration of externally injected electrons by the PBWA. ${ }^{9}$ The injected electron energy was $2 \mathrm{MeV}$ while $\gamma_{\mathrm{ph}}$ was 33 so the observation of electron energies of greater than $16.5 \mathrm{MeV}$ was conclusive proof that electrons had been trapped by the wave potential (Fig. 4). Maximum electron energies of $30 \mathrm{MeV}$ were observed implying an acceleration gradient of $\sim 2.8 \mathrm{GeV} / \mathrm{m}$ as the acceleration length was measured to be less than $1 \mathrm{~cm}$ long. Furthermore, loss of electron energy was seen as well as gain. This was to be expected as the wavelength of the plasma oscillation was $300 \mu \mathrm{m}$ (or 1 ps duration) whereas the injected electron microbunch was $10 \mathrm{ps}$ long. The data shown in Fig. 4 was obtained over many laser shots and as there was a $\pm 50 \mathrm{ps}$ jitter between the laser and the electron beam, the electron beam sampled plasma waves of greatly different amplitudes from one shot to the next. 


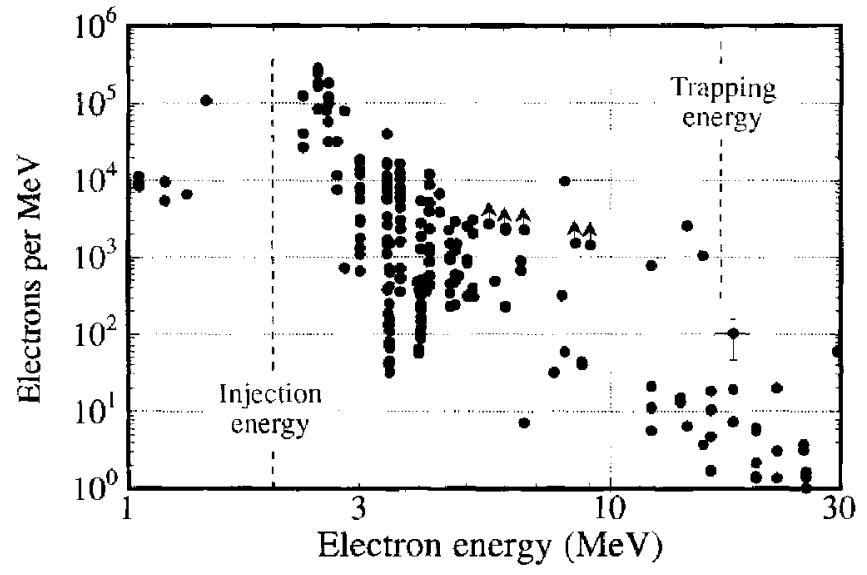

Figure 4. Trapping of externally injected electrons in the beat-excited relativistic plasma wave. (Ref.9)

It had taken 9 years from the time a relativistic plasma wave was first excited using the beat wave technique ${ }^{7}$ to do a convincing acceleration experiment ${ }^{8}$ mainly because the new Laser Acceleration field turned out to be cross-disciplinary requiring state-of-the-art expertise in lasers, particle accelerators and plasmas. In fact in 1994, the UCLA group proposed a $1 \mathrm{GeV}$ PBWA experiment ${ }^{10}$ based on then available $1 \mu \mathrm{m}$ CPA laser technology, but unfortunately such an experiment was never funded due to lack of resources.

The UCLA group subsequently proposed a more modest $100 \mathrm{MeV}$ PBWA experiment whose goal is to demonstrate acceleration of a significant number of electrons while maintaining a reasonable emittance $(<10 \pi \mathrm{mm} \mathrm{mrad})$ and energy spread $(<10 \% \Delta \gamma / \gamma)$. This is known as the Neptune project. This meant that a photoinjector linac with an extremely good beam quality had to be built as an injector ${ }^{11}$ and the $\mathrm{CO}_{2}$ laser had to be upgraded to give $1 \mathrm{TW}$ of peak power. ${ }^{12}$ The Neptune facility recently successfully finished its construction phase and the experimental team has begun the experiments on the $100 \mathrm{MeV}$ PBWA.

To complete the status report on the PBWA, other notable groups that have contributed in this field are those from U. Osaka (Japan), ${ }^{13}$ Imperial College (U.K.), ${ }^{14}$ Ecole Polytechnique (France) ${ }^{15}$ and Chalk River Laboratory of AEC, Canada. ${ }^{16}$ These experiments were carried out using both $1 \mu \mathrm{m}$ and $10 \mu \mathrm{m}$ laser pulses and have demonstrated acceleration of self-trapped ${ }^{13}$ as well as externally injected electrons. ${ }^{15,16}$

\section{STATUS REPORT ON THE SELF-MODULATED, LASER-WAKE FIELD ACCELERATION (SMLWFA)}

Now I will discuss the SMLWFA scheme. ${ }^{2}$ In the 1D manifestation of this scheme, the Raman Forward Instability (RFI) ${ }^{17}$ plays a critical role in establishing the 
relativistic plasma wave. In the RFI, the laser beam decays into a forward propagating Stokes wave, an anti-Stokes wave and a relativistic plasma wave. Once the Stokes and the anti-Stokes waves become sufficiently intense they beat with the pump wave to produce a deeply amplitude modulated envelope of the electric field. As in the PBWA scheme the modulation has a frequency of $\omega_{p}$ and wave number $k_{p}=k_{o}-k_{s}$ where "o" denotes the pump and "s "denotes the Stokes wave, respectively.

When the laser pulse is short compared to $Z_{R} / c$ where $Z_{R}$ is the Rayleigh length of the focused laser beam, the RFI is in the so-called spatio-temporal regime ${ }^{18}$ where the spatio-temporal gain $\mathrm{G}=\mathrm{e}^{\mathrm{g}} /(2 \pi \mathrm{g})^{1 / 2}$ with

$$
\mathrm{g}=\left(\mathrm{a}_{\mathrm{o}} / \sqrt{2}\right)\left(\omega_{\mathrm{p}} / \omega_{\mathrm{o}}\right)^{2}\left(\omega_{\mathrm{o}} / \mathrm{c}\right) \sqrt{(\mathrm{x}-\phi} / \phi
$$

Figure 5 shows the number of exponentiations of the growth $\mathrm{G}$ from an initial noise level of $\varepsilon_{\text {noise }} \sim 10^{-5}$ as the laser intensity (expressed in units of $a_{0}$ ) is varied for different densities assuming a $1 \mu \mathrm{m}$ laser. One can see that the number of e-foldings of growth increases first with $a_{0}$ but subsequently remains rather constant or even decreases for $a_{0}>1$ ). On the other hand, $\operatorname{Ln}(G)$ is a strong function of plasma density. There are only 2 e-foldings of growth when $\mathrm{n}=5 \times 10^{18} \mathrm{~cm}^{-3}$ but this number approaches 12 when the density increases to $1.5 \times 10^{19} \mathrm{~cm}^{-3}$. Thus, at these high densities we expect to see self-trapping of background plasma electrons and indeed wave breaking ${ }^{19}$ of the plasma oscillation.

The first paper that pointed out the RFI's role in electron acceleration was by C. Joshi et al. ${ }^{20}$ who used a relatively long but intense $\mathrm{CO}_{2}$ laser pulse $\left(\mathrm{a}_{\mathrm{o}} \sim 0.3, \omega / \omega_{\mathrm{p}}\right.$ $\sim 2.2$ ) to form a thin foil carbon plasma and measured the spectrum of forward and

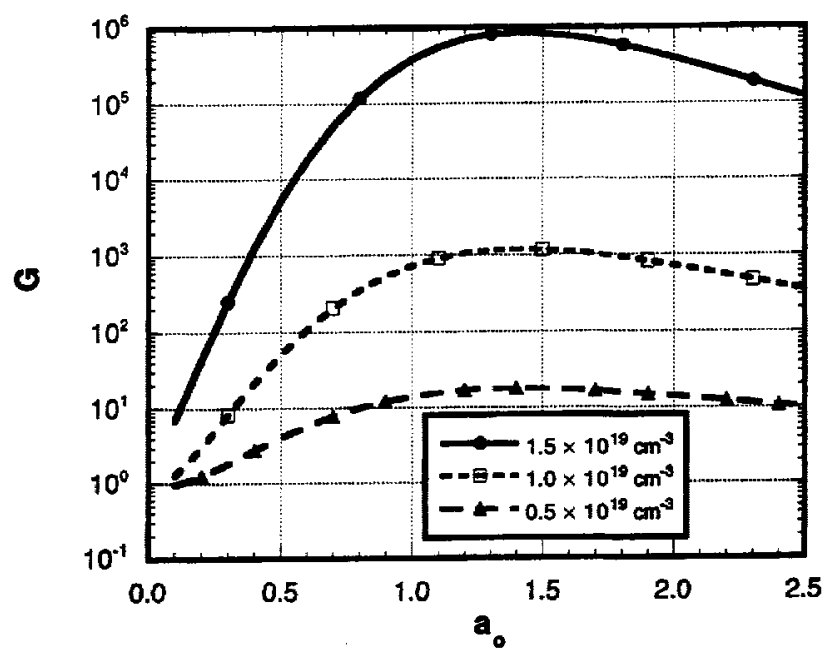

Figure 5. Spatio-temporal growth rate for RFI as a function density and laser power for a $1 \mu \mathrm{m}$ laser, nominally $800 \mathrm{fs}$ long (Ref. 19). 
backward emitted electrons. (Figure 6) Electrons with higher energies (up to 1.4 $\mathrm{MeV}$ ) were emitted in the forward direction compared with up to $0.8 \mathrm{MeV}$ in the backward direction and were attributed to RFI. Electrons were observed without external injection which means that they came from self-trapping of plasma electrons.

It was not until 1995 that the research on SMLWFA scheme began in earnest. The reason for this being very simple. The CPA technique ${ }^{21}$ had by then enabled many groups to have access to TW class $1 \mu \mathrm{m}$ lasers which were essential for this scheme. In 1993, the LLNL and the UCLA group jointly showed acceleration of electrons via the RFI conclusively using a $1 \mu \mathrm{m}$ laser. ${ }^{22}$ In the RFI, the single frequency pump laser decays into a comb of Stokes and anti-Stokes side bands each frequency shifted by $\omega_{p}$. In this experiment, the first two anti-Stokes side bands were seen. The amplitude of the first anti-Stokes wave was found to correlate well with the number of energetic electrons observed in the forward direction. Electrons up to $2 \mathrm{MeV}$ energy were seen when an $a_{o}=0.9$ laser was used to produce and interact with a $10^{19} \mathrm{~cm}^{-3}$ gas jet plasma.

The Livermore-UCLA experiment ${ }^{22}$ was soon followed by a much more sophisticated experiment at the Rutherford Appleton Laboratory ${ }^{23}$ in the U.K. by the Imperial College, UCLA and Ecole Polytechnique groups. The $1 \mu \mathrm{m}$ Vulcan laser at the laser facility had the capability of delivering up to $30 \mathrm{TW}$ of power in an $800 \mathrm{fs}$ pulse. Using this laser $\left(a_{0} \geq 2\right)$ and a gas-jet plasma target this group was able to see copious fast electron generation via breaking of the Raman Forward plasma wave. The evidence for wave breaking came from the sudden broadening of the comb of satellites in the forward direction (See Fig. 7) as the plasma density was increased

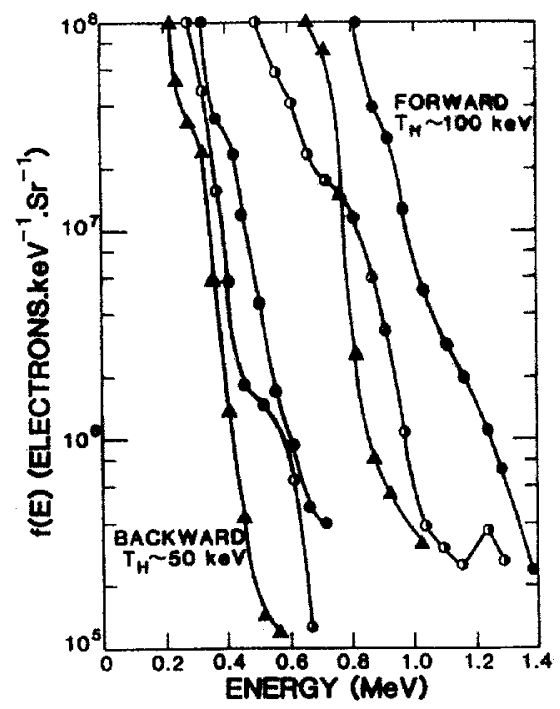

Figure 6. Forward and backward emitted electron spectrum from thin carbon foil plasma irradiated by an intense $\mathrm{CO}_{2}$ laser pulse (Ref. 20). 


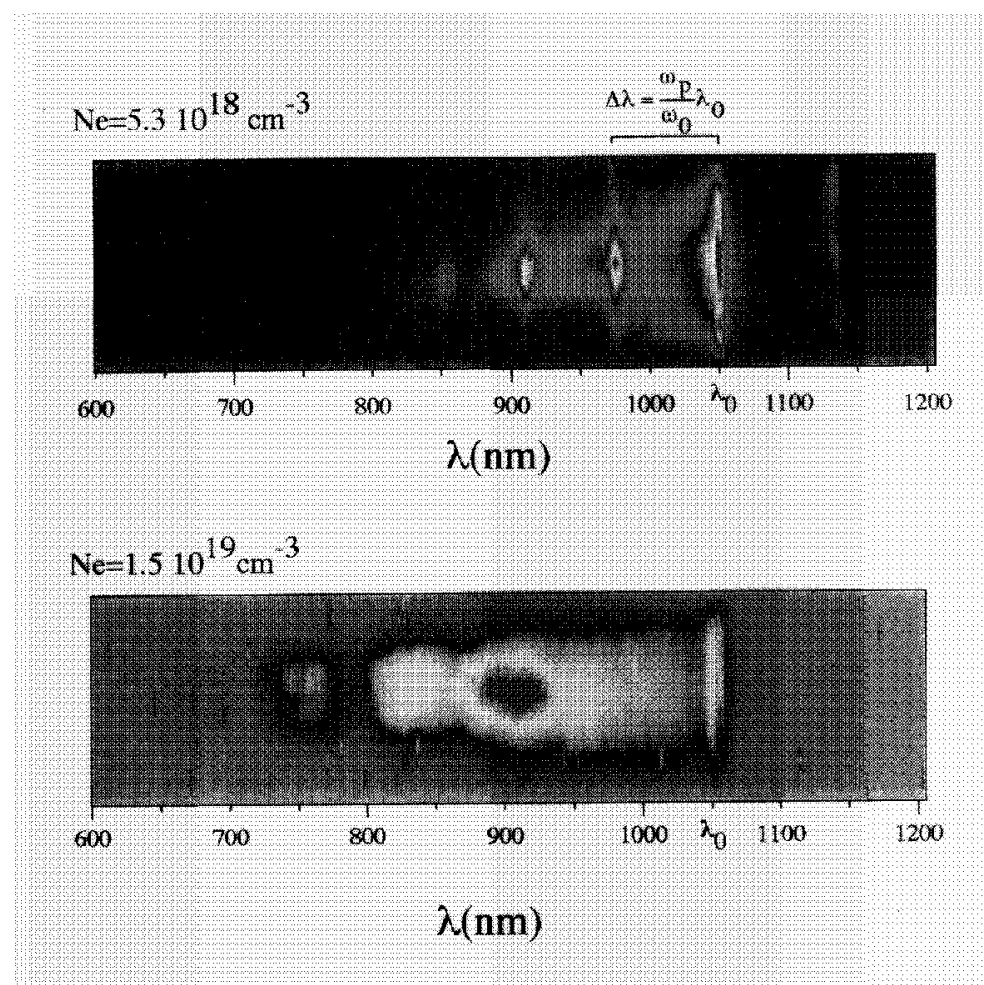

Figure 7. Spectrum of satellites to the pump laser in the forward direction at two different densities in the Rutherford experiment (Ref. 23).

from $5.3 \times 10^{18} \mathrm{~cm}^{-3}$ to $1.5 \times 10^{19} \mathrm{~cm}^{-3}$. The spatial extent of the relativistic plasma wave was also directly measured using Thomson scattering of a probe beam ${ }^{24}$ as was the spectrum of the relativistic electrons that were escaping the plasma in the forward direction (Fig. 8). From the maximum observed energy of $94 \mathrm{MeV}$ the accelerating gradient was deduced to be greater than $150 \mathrm{GeV} / \mathrm{m}$ which represents a record of sorts for the highest gradient terrestrial acceleration of charged particles. Another interesting aspect of this experiment is that the maximum energies observed were greater than those expected from the phase slippage between the electrons and the accelerating electric field of the plasma wave as given by the linear theory for preinjected electrons.

Many other groups around the world soon experimented with the SMLWFA scheme. Most notably the U. Michigan group ${ }^{25}$ and the NRL group ${ }^{26}$ both observed the anti-Stokes side bands and electrons. The Michigan group showed that the electrons were emitted in a well-defined beam ${ }^{25}$ in the same direction as the laser. A result from the NRL group is shown in Fig. 9 which qualitatively looks similar to the Rutherford results however, what is surprising about the NRL data is that the electron spectrum was found to extend out to $100 \mathrm{MeV}$ at a much lower peak laser power $(\sim 2$ TW), whereas Rutherford experiments were carried out at around $25 \mathrm{TW}$. The NRL 

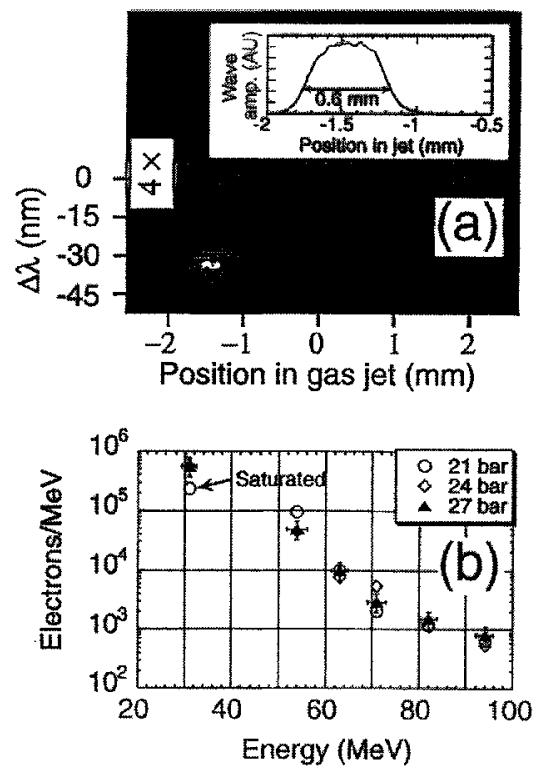

Figure 8. (a) transverse, small angle Thomson scattering showing the spatial extent of the RFS plasma wave and (b) the electron spectrum emitted in the forward direction in the Rutherford experiments (Ref. 20).

group did coherent Thomson scattering measurements on the plasma wave and determined that it lasts for about 30 ps or roughly 100 oscillations before decaying into ion acoustic waves.

An interesting byproduct of the SMLWFA experiments is the observation of relativistic self-focusing and filamentation of the laser beam in the plasma. This is so because the threshold for both Raman Forward and relativistic self-focusing are about the same for $n_{c} / n_{e}$ of about 100 . In the Rutherford experiments ${ }^{27}$ where the ratio of $P / P_{c}$ was about 20 where $P_{c}$ is the critical power for whole beam relativistic guiding, a relativistic plasma wave that was about 24 Rayleigh lengths long was observed and presumed to be inside a filament of similar length observed by imaging sidescattered light. The observation of the plasma wave puts a lower bound on the intensity of light inside the filament (Fig. 10) to be around $10^{18} \mathrm{~W} / \mathrm{cm}^{-2}$. Experiments at U. Michigan ${ }^{28}$ observed the onset of relativistic guiding very close to the theoretically predicted threshold. Furthermore, when a preformed channel with a density minimum on axis was produced using a radially expanding plasma column, this group showed that a second probe pulse could be guided by this plasma fiber. ${ }^{29}$ Experiments on preformed plasma fibers have been pioneered by the Maryland group ${ }^{30}$ and reproduced in various forms by others such as at $\mathrm{U}$. Texas, ${ }^{31} \mathrm{LBL}^{32} \mathrm{NRL}^{33}$ and elsewhere and peak laser intensities of $\sim 2 \times 10^{17} \mathrm{~W} / \mathrm{cm}^{2}$ have been transported over $\sim 20$ Rayleigh lengths. 


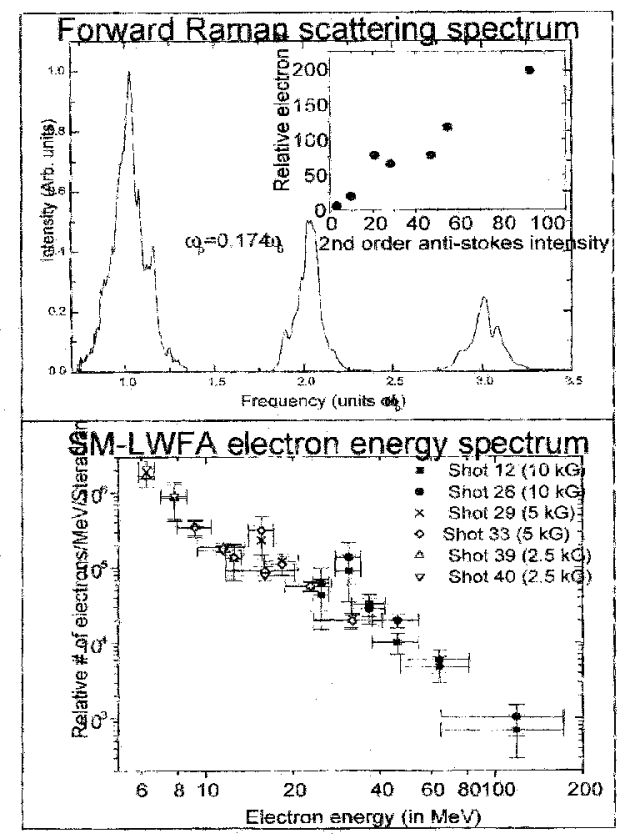

Figure 9.(a) Forward emitted anti-Sokes side bands in the NRL experiments and the linear relationship between number of accelerated electrons with the amplitude of the second anti-Stokes (inset; b) the accumulated electron energy spectrum in the forward direction in the NRL-SMLWFA experiments.
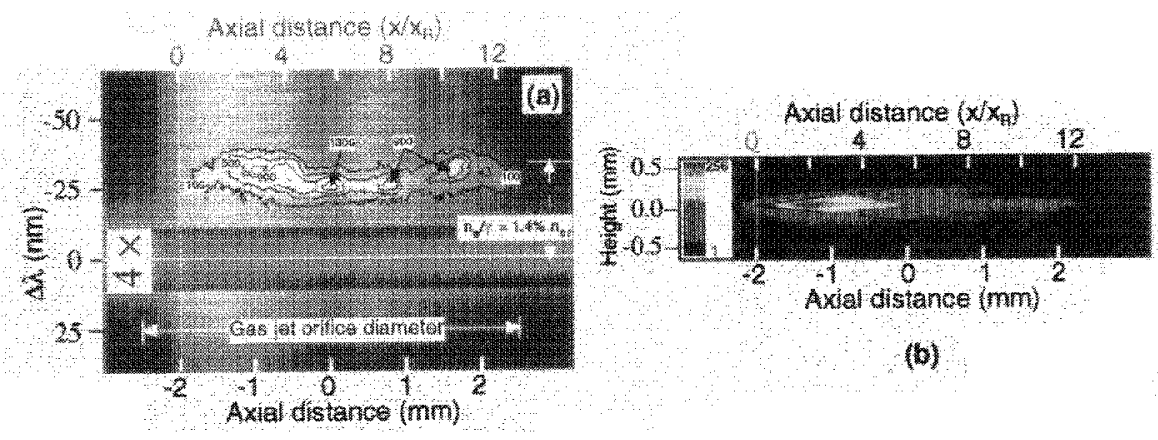

(b)

Figure 10. (a) Frequency-resolved image of EPW amplitude along the laser propagation axis. Contours are of constant scattered probe energy and are artificially suppressed at the edges relative to $x=0$ due to the temporal profile of the probe pulse. Spatial-modulated Bremsstrahlung continuum is also apparent. (b) Side-view sidescatter near $1 \mu \mathrm{m}$ for the same shot (color). 
An interesting variation on the laser guiding experiments is being attempted at NRL and Ecole Polytechnique. This is laser pulse propagation through thin capillary tubes. $^{34}$ In the experiment at NRL for instance, an electrical discharge through a $2 \mathrm{~cm}$ long capillary generates a density minimum on axis. A laser beam with a diameter of $\sim 35 \mu \mathrm{m}$ and a peak intensity of $10^{17} \mathrm{~W} / \mathrm{cm}^{2}$ is found to be guided over 22 Rayleigh lengths with an energy transfer efficiency of greater than $70 \%$ with an excellent mode structure. These experiments are significant and important because if the laser intensity transported could be increased to $\sim 10^{18} \mathrm{~W} / \mathrm{cm}^{2}$ over about $1 \mathrm{~cm}$ then a $1 \mathrm{GeV}$ SMLWFA suddenly becomes realizable.

\section{STATUS REPORT ON THE LASER WAKE FIELD ACCELERATION (LWFA) EXPERIMENTS}

In the LWFA a short $\left(\tau \sim \omega_{\mathrm{p}} / \mathrm{c}\right)$ but intense $\left(\mathrm{a}_{\mathrm{o}} \sim 1\right)$ laser pulse sent through a plasma leaves behind it a "wake" of plasma oscillation which has a phase velocity $\mathrm{v}_{\phi}$ which is equal to the group velocity $\mathrm{v}_{\mathrm{g}}$ of the laser pulse in the plasma. For $\omega_{0} \gg$ $\omega_{\mathrm{p}}, \mathrm{v}_{\mathrm{g}} \cong \mathrm{c}$ and the resultant plasma wake is relativistic. This was the original scheme proposed by T. Tajima and J. Dawson. ${ }^{2}$ The scheme was however not shown to work as the required laser pulses $\left(<100 \mathrm{fs}\right.$ in duration with focused intensities of $>10^{18}$ $\mathrm{W} / \mathrm{cm}^{2}$ for $\lambda<1 \mu \mathrm{m}$ ) did not exist until the Ti-saphire revolution in the late 1990's.

The first observation of a wake produced by a single short laser pulse was in 1996 by the Ecole Polytechnique ${ }^{35,36}$ and by the U.T. Austin groups. ${ }^{37}$ In both of these experiments the laser was focused to a spot size much smaller than wavelength of the plasma oscillation and consequently, the oscillation was dominated mainly by the radial motion of the electrons. Such cylindrical electron wakes were measured, with a temporal resolution much better than $\omega_{\mathrm{p}} / \mathrm{c}$ by frequency domain interferometry.

In the Ecole Polytechnique experiments, the cylindrical plasma wake field excited by a $130 \mathrm{fs}$ Ti-saphire laser was seen to have a nonlinear increase in oscillation frequency as the plasma density was decreased below the optimum density. The plasma wave was also seen to damp in a few plasma periods. ${ }^{35}$ These experiments were followed up by a proof-of-principle acceleration experiment by the same group. ${ }^{38}$ By injecting a $3 \mathrm{MeV}$ electron beam into the wake a maximum energy gain of 1.6 $\mathrm{MeV}$ was measured, corresponding to a maximum longitudinal field of $1.5 \mathrm{GeV} / \mathrm{m}$. Experiments on LWFA are in progress at JERI/KEK laboratories with published data on acceleration that has caused much excitement and controversy.

Finally, the IC group, ${ }^{39}$ the LBL group, ${ }^{40}$ and the U. Michigan group ${ }^{41}$ are all beginning to use nuclear activation technique as a method for characterizing the flux of electrons above a certain threshold energy in their respective experiments. It is quite possible that the first use of these laser electron accelerators may well turn out to be for generating rare radioactive isotopes for specialized uses. 


\section{ACKNOWLEDGMENTS}

I am grateful to Mike Downer, Tony Ting, J. Wurtele, D. Umstadter, W. Leemans, and Zulfikar Najmudin for providing me with information on their latest research.

\section{REFERENCES}

1. C. Joshi et al., Nature 311, 525 (1984).

2. P. Sprangle et al., Phys. Rev. Lett. 72,2887 (1994).

3. T. Tajima and J. M. Dawson, Phys. Rev. Lett. 43, 267 (1979).

4. T. Coffey, Phys. Fluids 14, 1402 (1971).

5. R. Williams et al., Laser and Particle Beams 8(3), 427 (1990).

6. M. N. Rosenbluth and C. S. Liu, Phys. Rev. Lett. 29, 701 (1972).

7. C. Clayton et al., Phys. Rev. Lett. 54,2343(1985).

8. C. Clayton et al., Phys. Rev. Lett. 71, 37(1993).

9. M. Everett et al., Nature 368, 527 (1994).

10. C. Joshi et al., Comments on Plasma Phys. and Nucl. Fusion, 16, 65 (1994).

11. J. Rosenzweig et al., Nucl. Intsr. Meth. A. 410, 437 (1998).

12. S. Tochitsky et al., Optics Lett. 24, 1717 (1999).

13. Y. Kitagawa et al., Phys. Rev. Lett. 68, 48 (1992).

14. A. Dyson et al., Plasma Phys. Controlled Fusion, 38, 509 (1996).

15. F. Amiranoff et al., Phys. Rev. Lett. 68, 3710 (1992).

16. N. A. Ebrahim, J. Appl. Phys. 76, 7645 (1994).

17. D. W. Forslund et al., Physics Fluids 18, 1002 (1075).

18. W. Mori et al., Phys. Rev. Lett. 72, 1482 (1994).

19. J. M. Dawson, Phys. Fluids 113, 383 (1959).

20. C. Joshi et al., Phys. Rev. Lett. 47, 1285 (1981).

21. P. Maine et al., IEEE J. Q. E. 24, 398 (1988).

22. C. Coverdale et al., Phys. Rev. Lett. 74, 4659 (1998).

23. A Modena et al., Nature 377,606 (1995).

24. D. Gordon et al., Phys. Rev. Lett. 80, 2133 (1998).

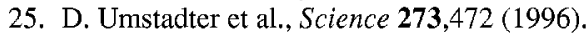

26. A. Ting et al., Phys. Rev. Lett.77, 5377 (1996).

27. C. Clayton et al., Phys. Rev. Lett. 81, 100 (1998).

28. R. Wagner et al., Phys. Rev. Lett. 78, 3125 (1997).

29. S. Y. Chen et al., Phys. Rev. Lett. 80, 2610 (1998).

30. C. Durfee et al., Phys. Rev. Lett. 71, 2409 (1993).

31. G. Gaul et al., Proc. Int'l. Conf. LASERS'99, Quebe City, 13-16 Dec. (1999).

32. Volfbeyn et al., Phys. Plasmas 4, 3403 (1997).

33. C. Krushelnick et al., Phys. Rev. Lett. 78, 4047 (1997).

34. Y. Ehrlich et al., Phys. Rev. Lett. 77, 4186 (1996); F. Dorchies et al., Phys. Rev. Lett. 82, 4655 (1999).

35. J. R. Marqués et al., Phys. Rev. Lett.78, 3463 (1997).

36. J. R. Marqués et al., Phys. Rev. Lett. 76, 3570 (1996).

37. C. W. Siders et al., Phys. Rev. Lett. 76, 3370 (1996).

38. F. Amiranoff et al., Phys. Rev. Lett. 81, 995 (1998).

39. Z. Najmudin, Private Communication.

40. W. Leemans, at this meeting, see proceedings.

41. D. Umstadter, at this meeting, see proceedings. 